\title{
Construction and classification of complex simple Lie algebras via projective geometry
}

\author{
J.M. Landsberg and Laurent Manivel
}

\begin{abstract}
We construct the complex simple Lie algebras using elementary algebraic geometry. We use our construction to obtain a new proof of the classification of complex simple Lie algebras that does not appeal to the classification of root systems.
\end{abstract}

\section{Overview}

We first present an algorithm that constructs the minuscule varieties using elementary algebraic geometry. The minuscule varieties are a preferred class of homogeneous varieties. They are essentially the homogeneous projective varieties that admit an irreducible Hermitian symmetric metric; see below for the precise definition. The algorithm proceeds iteratively by building a larger space $X \subset \mathbb{P}^{N}$ from a smaller space $Y \subset \mathbb{P}^{n}$ via a rational map $\mathbb{P}^{n} \rightarrow \mathbb{P}^{N}$, defined using the ideals of the secant varieties of $Y$, beginning with $Y=\mathbb{C P}^{1}$. As a byproduct, we obtain elementary constructions of all complex simple Lie algebras (except for $\mathfrak{e}_{8}$ which has no minuscule homogenous space) and their minuscule representations, without any reference to Lie groups or Lie algebras.

Next we present an algorithm that constructs the fundamental adjoint varieties using the ideals of the tangential and secant varieties of certain minuscule varieties. By an adjoint variety, we mean the unique closed orbit in the projectivization $\mathbb{P} \mathfrak{g}$ of a simple complex Lie algebra $\mathfrak{g}$. We say that an adjoint variety is fundamental if the adjoint representation is fundamental. In particular, we construct all complex simple Lie algebras without any reference to Lie theory.

Complex simple Lie algebras were first classified by Cartan and Killing 100 years ago. Their classification proof proceeds by reducing the question to a combinatorial problem: the classification of irreducible root systems, and then classifying root systems.

We present a new proof of the classification of minuscule varieties and complex simple Lie algebras by showing our algorithms produce all minuscule (resp. fundamental adjoint) varieties without using the classification of root systems, although we do use some properties of root systems. We also provide a proof that the only non-fundamental adjoint varieties are the adjoint varieties of $A_{m}$ and $C_{m}$, and thus we obtain a new proof of the classification of complex simple Lie algebras.

Our proof can be translated into a combinatorial argument: the construction consists of two sets of rules for adding new nodes to marked Dynkin diagrams. As a combinatorial algorithm, it is less efficient than the standard proof, which proceeds by ruling out all but a short list of Dynkin diagrams immediately, and then studying the few remaining diagrams to see which are actually admissible. 
Our constructions have applications that go well beyond the classification proof presented in this article. This is the second paper in a series. In [8], [9] and [10] we present geometric and representation-theoretic applications of our algorithms. In [8] we study the geometry of the exceptional homogeneous spaces using the constructions of this paper. In [9] and [10] we apply the results of this paper, especially our observations about the Casimir in section 5 , to obtain decomposition and dimension formulas for tensor powers of some preferred representations.

\section{Statements of main results}

Let $V$ be a complex vector space and let $X \subseteq \mathbb{P} V$ be a variety in the associated projective space. Let $v_{d}(X) \subset \mathbb{P}\left(S^{d} V\right)$ denote its $d$-th Veronese re-embedding. If $P_{1}, \ldots, P_{N}$ is a basis of $S^{d} V^{*}$, the space of homogeneous polynomials of degree $d$ on $V$, then the map $\mathbb{P} V \rightarrow \mathbb{P}\left(S^{d} V\right)$ is $[x] \mapsto\left[P_{1}(x), \ldots, P_{N}(x)\right]$.

If $X \subseteq \mathbb{P} V$ and $Y \subseteq \mathbb{P} W$, we let $\operatorname{Seg}(X \times Y) \subset \mathbb{P}(V \otimes W)$ denote their Segre product, given by $([x],[y]) \mapsto[x \otimes y]$. The Segre product generalizes to an arbitrary number of factors.

We will use the notation $\langle X\rangle \subset \mathbb{P} V$ to denote the linear span of $X$.

Definition 2.1. Call a variety $X \subset \mathbb{P} V$ a minuscule variety if $X=G / P_{\alpha}$ where $G$ is a complex simple Lie group, $\alpha$ is a minuscule root, $P_{\alpha}$ is an associated maximal parabolic subgroup and $X$ is the projectivized orbit of a highest weight vector in $V=V_{\omega}$ where $\omega$ is the fundamental weight dual to the coroot of $\alpha$ (so the embedding is the minimal equivariant embedding). Call $X$ a generalized minuscule variety if $X$ is a Segre product of (Veronese re-embeddings of) minuscule varieties. In this situation we will call $V$ a minuscule (resp. generalized minuscule) $G$-module.

The generalized minuscule varieties are those varieties admitting a Hermitian symmetric metric induced from a Fubini-Study metric on the ambient projective space. The minuscule varieties are those for which the metric is irreducible and the embedding is minimal (i.e., not a Veronese re-embedding).

Definition 2.2. For a smooth variety $X \subset \mathbb{P} V$, let $\mathcal{T}(X) \subset G(2, V) \subset \mathbb{P}\left(\Lambda^{2} V\right)$ denote the variety of embedded tangent lines of $X$.

Let $\tau(X) \subset \mathbb{P} V$ denote the tangential variety of $X$ (the union of the points on embedded tangent lines), and let $\sigma_{p}(X) \subset \mathbb{P} V$ denote the variety of secant $\mathbb{P}^{p-1}$ 's to $X$, that is, for $x^{1}, \ldots, x^{p} \subset \mathbb{P} V$, let $\mathbb{P}_{x^{1}, \ldots, x^{p}}$ denote the projective space they span (generally a $\mathbb{P}^{p-1}$ ), then $\sigma_{p}(X)=\overline{\cup_{x^{1}, \ldots, x^{p}} \in X^{\mathbb{P}} \mathbb{P}^{1}, \ldots, x^{p}}$. We let $\sigma(X)=\sigma_{2}(X)$.

\subsection{Minuscule case}

The minuscule algorithm. Let $Y=\operatorname{Seg}\left(v_{d_{1}}\left(X_{1}\right) \times \cdots \times v_{d_{r}}\left(X_{r}\right)\right) \subset \mathbb{P}^{n-1}=\mathbb{P} T$ where the $X_{j} \subseteq \mathbb{P}^{N_{j}}$ 's are outputs of previous runs through the algorithm or $\mathbb{P}^{1} \subseteq \mathbb{P}^{1}$.

We will call $Y$ admissible if $\mathcal{T}(Y)$ is linearly nondegenerate, that is, if $\langle\mathcal{T}(Y)\rangle=\mathbb{P}\left(\Lambda^{2} T\right)$. If $Y$ is admissible, then define a rational map as follows: let $d$ be the positive integer such that $\sigma_{d-1}(Y) \neq \sigma_{d}(Y)=\mathbb{P}^{n-1}$. Linearly embed $\mathbb{P}^{n-1} \subset \mathbb{P}^{n}$ as the hyperplane $\left\{x_{0}=0\right\}$, and consider the rational map

$$
\begin{aligned}
& \phi: \quad \mathbb{P}^{n} \quad \rightarrow \mathbb{P}^{N} \subset \mathbb{P}\left(S^{d} \mathbb{C}^{n+1 *}\right) \\
& {\left[x_{0}, \ldots, x_{n}\right] \mapsto\left[x_{0}^{d}, x_{0}^{d-1} T^{*}, x_{0}^{d-2} I_{2}(Y), x_{0}^{d-3} I_{3}\left(\sigma_{2}(Y)\right), \ldots, I_{d}\left(\sigma_{d-1}(Y)\right)\right],}
\end{aligned}
$$

and call $X=\phi\left(\mathbb{P}^{n}\right) \subset \mathbb{P}^{N}$ an output. Here $T^{*}$ and $I_{k}(Z)=I_{k}(Z, \mathbb{P} T)$ are shorthand notation respectively for a basis of $T^{*}$ and a set of generators of the ideal of $Z$ in degree $k$. 
Remark 2.3. We show below that the admissiblity hypothesis implies $r, d_{j} \leq 2$ and thus there is a finite number of varieties to test at each run through the algorithm. (Moreover, if $d_{1}=2$ then $r=1$.)

Theorem 2.4. (Geometric construction of minuscule varieties.) The minuscule varieties are exactly the outputs of the minuscule algorithm.

Theorem 2.5. (The minuscule algorithm is effective.) After six runs the minuscule algorithm stabilizes and one can determine all minuscule varieties from the output of the first six runs. In particular, the minuscule algorithm gives an effective classification of minuscule varieties.

\subsection{Adjoint case}

The adjoint algorithm. Let $Y \subset \mathbb{P}^{n-2}=\mathbb{P} T_{1}$ be a generalized minuscule variety. Here we define $Y$ to be admissible if $\langle\mathcal{T}(Y)\rangle \subseteq \mathbb{P}\left(\Lambda^{2} T_{1}\right)$ has codimension one. Note that admissibility implies there is an up to scale two-form defined on $T_{1}$. If $Y$ is admissible, define a rational map as follows: linearly embed $\mathbb{P}^{n-2} \subset \mathbb{P}^{n-1} \subset \mathbb{P}^{n}$ respectively as the hyperplanes $\left\{x_{n}=0\right\}$ and $\left\{x_{0}=0\right\}$, consider the rational map

$$
\begin{aligned}
& \phi: \quad \mathbb{P}^{n} \quad \rightarrow \mathbb{P}^{N} \subset \mathbb{P}\left(S^{4} \mathbb{C}^{n+1 *}\right) \\
& {\left[x_{0}, \ldots, x_{n}\right] \mapsto\left[x_{0}^{4}, x_{0}^{3} T_{1}^{*}, x_{0}^{3} x_{n}, x_{0}^{2} I_{2}(Y), x_{0}^{2} x_{n} T_{1}^{*}-x_{0} I_{3}\left(\tau(Y)_{\text {sing }}\right), x_{0}^{2} x_{n}^{2}-I_{4}(\tau(Y))\right],}
\end{aligned}
$$

and call $X=\phi\left(\mathbb{P}^{n}\right) \subset \mathbb{P}^{N}$ an output.

Note that to make sense of our notation in the mapping, we are using that $\tau(Y)$ is a quartic hypersurface, which is proved in $\S 6.1$. In particular $I_{3}\left(\tau(Y)_{s i n g}\right)$ is the space of derivatives of the equation of $\tau(Y)$.

Remark 2.6. We show below that the admissibility hypothesis implies that $d_{j}, r \leq 3$, so again, there are a finite number of cases to check and the algorithm is effective. (Moreoever $d_{1}=3$ implies $r=1$ and $d_{1}=2$ implies $r \leq 2$.)

Theorem 2.7. The fundamental adjoint varieties are exactly the varieties constructed by the adjoint algorithm.

Corollary 2.8. The fundamental adjoint varieties are not rigid to order two. More precisely, there exist varieties $X^{n} \subset \mathbb{P}^{N}$ with the same projective second fundamental form as a fundamental adjoint variety at a general point which are not fundamental adjoint varieties.

Proof. If we write the image of $\phi$ as a graph in local coordinates about $[1,0, \ldots, 0]$, the Taylor series will truncate after the fourth order term. If we take the same Taylor series and truncate after the second order term, we obtain, in this affine open subset, a variety with the same second fundamental form as $\phi\left(\mathbb{P}^{n}\right)$ at $[1,0, \ldots, 0]$ on the entire affine open subset, and this open variety can be completed to a projective variety. (In general, varieties written as graphs with only second order Taylor series have constant second fundamental forms in region where the coordinates are valid.) On the other hand, we show below that the image of $\phi$ is homogeneous and thus has the same second fundamental form at all points.

Remark 2.9. The corollary contrasts the case of the Severi varieties and other minuscule varieties of small codimension, which are rigid to order two; see [6]. 
Our classification proof will be complete once we prove that the only simple Lie algebras where the adjoint representation is not fundamental are $A_{m}$ and $C_{m}$. We prove this in $\S 6.3$.

Remark 2.10. In many ways, the exceptional groups are better behaved than the classical groups from the perspective of our constructions. For example:

- The only "exception" in the minuscule algorithm is the Grassmanian $X=G(k, V)$, constructed from $Y=\mathbb{P}^{k-1} \times \mathbb{P}^{l-1}$. In all other cases the $P$-module $\Lambda^{2} T$ contains no $P$ submodules and there is no need to study $\langle\mathcal{T}(Y)\rangle$.

- The only pathological (i.e., non-fundamental) adjoint varieties are those of $A_{m}, C_{m}$.

- For the minuscule algorithm, the admissible $Y$ 's which yield exceptional $X$ 's are such that $\operatorname{codim} \sigma(Y)$ is at most one, so the rational map $\phi$ is at most cubic.

\section{$3 \quad$ Examples}

\subsection{Minuscule case}

For the first run through the algorithm, the admissible varieties and their outputs are

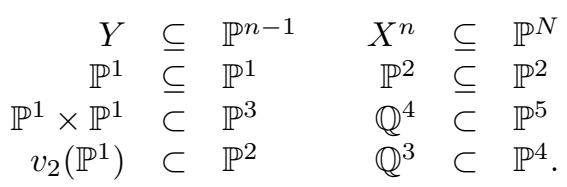

Here and below, $\mathbb{Q}^{m} \subset \mathbb{P}^{m+1}$ denotes the smooth quadric hypersurface.

For the second round,

$\begin{array}{rll}Y & \subseteq & \mathbb{P}^{n-1} \\ \mathbb{P}^{2} & \subseteq & \mathbb{P}^{2} \\ v_{2}\left(\mathbb{P}^{2}\right) & \subset & \mathbb{P}^{5} \\ \mathbb{P}^{1} \times \mathbb{P}^{2} & \subset & \mathbb{P}^{5} \\ \mathbb{P}^{2} \times \mathbb{P}^{2} & \subset & \mathbb{P}^{8} \\ \mathbb{Q}^{4} & \subset & \mathbb{P}^{5} \\ \mathbb{Q}^{3} & \subset & \mathbb{P}^{4}\end{array}$

$\begin{array}{rll}X^{n} & \subseteq & \mathbb{P}^{N} \\ \mathbb{P}^{3} & \subseteq & \mathbb{P}^{3} \\ G_{\text {Lag }}(3,6) & \subset & \mathbb{P}^{11} \\ G(2,5) & \subset & \mathbb{P}^{9} \\ G(3,6) & \subset & \mathbb{P}^{19} \\ \mathbb{Q}^{6} & \subset & \mathbb{P}^{8} \\ \mathbb{Q}^{5} & \subset & \mathbb{P}^{7} .\end{array}$

Here $G(k, l)$ denotes the Grassmanian of $k$-planes in $\mathbb{C}^{l}$ and $G_{\text {Lag }}(k, 2 k)$ denotes the Grassmanian of Lagrangian $k$-planes for a given symplectic form.

Continuing, one gets

$$
\begin{aligned}
& \begin{array}{rll}
Y & \subseteq & \mathbb{P}^{n-1} \\
\mathbb{P}^{n-1} & \subseteq & \mathbb{P}^{n-1}
\end{array} \\
& v_{2}\left(\mathbb{P}^{m-1}\right) \subset \mathbb{P}^{\left(\begin{array}{c}
m+1 \\
2
\end{array}\right)-1} \\
& \mathbb{P}^{k-1} \times \mathbb{P}^{l-1} \subset \mathbb{P}^{k l-1} \\
& \mathbb{Q}^{2 m-2} \subset \mathbb{P}^{2 m-1} \\
& \mathbb{Q}^{2 m-1} \subset \mathbb{P}^{2 m} \\
& G(2, m) \quad \subset \quad \mathbb{P}^{\left(\begin{array}{c}
m \\
2
\end{array}\right)-1}
\end{aligned}
$$

$$
\begin{aligned}
& \begin{aligned}
X^{n} & \subseteq \mathbb{P}^{N} \\
\mathbb{P}^{n} & \subseteq \mathbb{P}^{n}
\end{aligned} \\
& G_{\text {Lag }}(m, 2 m) \subset \mathbb{P}^{C_{m+1}-1} \\
& G(k, k+l) \quad \subset \mathbb{P}^{\left(\begin{array}{c}
k+l \\
k
\end{array}\right)-1} \\
& \mathbb{Q}^{2 m} \subset \mathbb{P}^{2 m+1} \\
& \mathbb{Q}^{2 m+1} \subset \mathbb{P}^{2 m+2} \\
& \mathbb{S}_{m} \subset \mathbb{P}^{2^{m-1}-1} .
\end{aligned}
$$

Here $C_{m+1}=\frac{1}{m+2}\left(\begin{array}{c}2 m+2 \\ m+1\end{array}\right)$ is the $(m+1)$-st Catalan number, and the spinor variety $\mathbb{S}_{m}$ of $D_{m}$ consists of one family of maximal isotropic subspaces of $\mathbb{C}^{2 m}$ endowed with a nondegenerate quadratic form and embedded in the projectivization of one of the two halfspin representations. 
Continuing, one sees that $G_{L a g}(m, 2 m)$ and $\mathbb{S}_{m}$ are terminal except for $\mathbb{S}_{5}$ which yields two exceptional spaces:

$$
\begin{array}{rrrrrr}
Y & \subseteq & \mathbb{P}^{n-1} & X^{n} & \subseteq & \mathbb{P}^{N} \\
\mathbb{S}_{5} & \subset & \mathbb{P}^{15} & \mathbb{O P}^{2} & \subset & \mathbb{P}^{26} \\
\mathbb{O P}^{2} & \subset & \mathbb{P}^{26} & G_{w}\left(\mathbb{O}^{3}, \mathbb{O}^{6}\right) & \subset & \mathbb{P}^{55}
\end{array}
$$

Here $\mathbb{O P}^{2}$ denotes the sixteen dimensional Cayley plane, which is the 16 -dimensional homogeneous variety of $E_{6}$, and $G_{w}\left(\mathbb{O}^{3}, \mathbb{O}^{6}\right)$ denotes the 27 -dimensional minuscule variety of $E_{7}$.

Proof of 2.5. First observe that $\langle\mathcal{T}(Y)\rangle \neq \mathbb{P}\left(\Lambda^{2} T\right)$ for the Segre product of three projective spaces. In fact, for $Y=\operatorname{Seg}\left(\mathbb{P}^{1} \times \mathbb{P}^{1} \times \mathbb{P}^{1}\right), \operatorname{codim}\langle\mathcal{T}(Y)\rangle=1\left(\right.$ and $Y=\operatorname{Seg}\left(\mathbb{P}^{1} \times \mathbb{P}^{1} \times \mathbb{P}^{1}\right)$ yields the $D_{4}$ adjoint variety), and $\operatorname{codim}\langle\mathcal{T}(Y)\rangle>1$ for all others. Thus one cannot have a triple Segre product $\operatorname{Seg}\left(Y_{1} \times Y_{2} \times Y_{3}\right)$ for any $Y_{j}$ in the minuscule algorithm, and for any $Y_{j}$ 's other than three $\mathbb{P}^{1}$ 's in the adjoint algorithm.

For the Segre product of two varieties $\operatorname{Seg}\left(Y_{1} \times Y_{2}\right) \subset \mathbb{P}\left(W_{1} \otimes W_{2}\right), \Lambda^{2} W_{1} \otimes I_{2}\left(Y_{2}\right)$ will not be in $\langle\mathcal{T}(Y)\rangle$ and similarly with roles reversed. (The ideals of homogeneous varieties are generated in degree two; see [11.) So only a double product of projective spaces is admissible for the first algorithm and in the second algorithm, only $\mathbb{P}^{1} \times \mathbb{Q}^{m}$ is possible.

For a Veronese embedding of a projective space, only $Y=v_{2}\left(\mathbb{P}^{m}\right)$ has $\mathcal{T}(Y)$ linearly full, and only $v_{3}\left(\mathbb{P}^{1}\right)$ has $\langle\mathcal{T}(Y)\rangle$ of codimension one, so no Veronese re-embedding of a subvariety of $\mathbb{P}^{m}$ will be admissible for either algorithm.

Thus, other than the examples mentioned above, the only new inputs to the minuscule algorithm must be outputs from a previous round. From the list of examples above, we see that the algorithm stabilizes after the sixth iteration.

\subsection{Adjoint case}

The generalized minuscule varieties yielding fundamental adjoint varieties are

$$
\begin{array}{rlll}
Y & \subset & \mathbb{P}^{n-2} & G \\
v_{3}\left(\mathbb{P}^{1}\right) & \subset & \mathbb{P}^{3} & G_{2} \\
\mathbb{P}^{1} \times \mathbb{Q}^{2 m-3} & \subset & \mathbb{P}^{4 m-3} & B_{m} \\
\mathbb{P}^{1} \times \mathbb{Q}^{2 m-4} & \subset & \mathbb{P}^{4 m-5} & D_{m} \\
G_{\text {Lag }}(3,6) & \subset & \mathbb{P}^{13} & F_{4} \\
G(3,6) & \subset & \mathbb{P}^{19} & E_{6} \\
\mathbb{S}_{6} & \subset & \mathbb{P}^{31} & E_{7} \\
G_{w}\left(\mathbb{O}^{3}, \mathbb{O}^{6}\right) & \subset & \mathbb{P}^{55} & E_{8} .
\end{array}
$$

The two exceptional (i.e., non-fundamental) cases are

$$
\begin{array}{rllll}
\mathbb{P}^{k-1} \sqcup \mathbb{P}^{k-1} & \subset & \mathbb{P}^{2 k-1} & A_{k} \\
\emptyset & \subset & \mathbb{P}^{2 m-1} & C_{m} .
\end{array}
$$

Remark 3.1. From the examples one sees that the admissibility hypothesis for the minuscule algorithm is equivalent to requiring that $\tau(Y)=\sigma(Y)$ and admissibility for the adjoint algorithm is equivalent to requiring that $\tau(Y) \neq \sigma(Y)=\mathbb{P} T$. Although it turns out to be easier to use $\mathcal{T}(Y)$, our constructions were motivated by two theorems regarding secant and tangential varieties: the Fulton-Hansen theorem [3] and Zak's theorem on Severi varieties [13]. The construction in the minuscule algorithm is a generalization of Zak's construction of the Severi varieties. 


\section{Local differential geometry}

In this section, we characterize the generalized minuscule varieties $G / P$ defined in $\S 2$ in terms of local differential geometry. We choose a Borel subgroup $B$ of $G$ and a maximal torus $T$ of $B$, yielding a root system of the Lie algebra $\mathfrak{g}$, with a base of the simple roots. We say that a simple root $\alpha$ is short if there exists a longer root inside the root system of $\mathfrak{g}$. Up to conjugation, there is a natural correspondence between simple roots $\alpha$, maximal parabolic subgroups $P_{\alpha}$ and nodes of the Dynkin diagram of $G$. We begin with general considerations.

Let $X^{n} \subset \mathbb{P} V$ be any variety and let $x \in X$ be a smooth point. Let $\hat{T}_{x}{ }^{(k)} X \subseteq V$ denote the cone over the $k$-th osculating space and let $N_{k}=\hat{T}^{(k)} / \hat{T}^{(k-1)}$ denote the (twisted) $k$-th normal space of $X$ at $x$ (see [7], §2.1). If we choose local coordinates $\left(x^{1}, \ldots, x^{N}\right)$ at $x$ on $\mathbb{P} V$, adapted to the filtration by osculating spaces, we may write $X$ locally as a graph. Taking $\frac{\partial}{\partial x^{\alpha}}$ as a basis of $T_{x} X$, for $1 \leq \alpha \leq n$, we have in these coordinates, for $\operatorname{dim} \hat{T}^{(k-1)}<\mu_{k} \leq \operatorname{dim} \hat{T}^{(k)}$, the Taylor expansion:

$$
\begin{aligned}
x^{\mu_{2}} & =q_{\alpha \beta}^{\mu_{2}} x^{\alpha} x^{\beta}+r_{\alpha \beta \gamma}^{\mu_{2}} x^{\alpha} x^{\beta} x^{\gamma}+\ldots \\
x^{\mu_{3}} & =q_{\alpha \beta \gamma}^{\mu_{3}} x^{\alpha} x^{\beta} x^{\gamma}+r_{\alpha \beta \gamma \delta}^{\mu_{3}} x^{\alpha} x^{\beta} x^{\gamma} x^{\delta}+\ldots \\
& \vdots
\end{aligned}
$$

with summations over repeated indices. The projective differential invariants of $X$ at $x$ are as follows: the fundamental forms $\mathbb{F F}^{k}=\mathbb{F F}_{X, x}^{k}=F_{k+0, X, x}^{k} \in S^{k} T^{*} \otimes N_{k}$ are given by

$$
\mathbb{F F}^{k}=q_{\alpha_{1} \ldots \alpha_{k}}^{\mu_{k}} d x^{\alpha_{1}} \circ \ldots \circ d x^{a_{k}} \otimes \frac{\partial}{\partial x^{\mu_{k}}},
$$

and the relative differential invariants $F_{k+l, X}^{k}$ are given by

$$
F_{k+l, X, x}^{k}=r_{\alpha_{1} \ldots \alpha_{k+l}}^{\mu_{k}} d x^{\alpha_{1}} \circ \ldots \circ d x^{\alpha_{k+l}} \otimes \frac{\partial}{\partial x^{\mu_{k}}},
$$

where the $r_{\alpha_{1} \ldots \alpha_{k+l}}^{\mu_{k}}$ are the coefficients of the terms of degre $k+l$ in the Taylor expansion of $x^{\mu_{k}}$ (see [4 for more precise definitions). Note that these are a complete set of differential invariants in the sense that one can recover the Taylor series of $X$ at $x$ from them.

We use the notation $F_{k}=F_{k, X, x}^{2}, I I=\mathbb{F F}_{X, x}^{2},|I I|=\mathbb{F F}_{X}^{2}\left(N^{*}\right) \subseteq S^{2} T_{x}^{*} X$, and Base $|I I|=\{[v] \in \mathbb{P} T \mid I I(v, v)=0\}$.

For any variety, $F_{k+l, X}^{k}$ occurs as the coefficient of $\mathbb{F F}_{v_{l+1}(X)}^{k+l}$ in the direction of $N_{k} \circ(\hat{x})^{l}$ (with respect to the induced adapted framing); see 四, 3.10.

Example. Consider the cubic form $F_{3}=F_{3, X}^{2} . F_{3}(U v, Y v, Z v)$ is given by the component of $U Y Z v$ in $U(\mathfrak{g})_{2} v \cap N_{2}$, that is, the component of $U Y Z v$ in $U(\mathfrak{g})_{2} v$. Thus $F_{3}$ consists of sums of terms $U Y Z v$ such that $U Y Z v=\Sigma^{j} W_{1}^{j} W_{2}^{j} v$ for some $W_{1}^{j}, W_{2}^{j} \in \mathfrak{g}$. For example, if there are relations in $U(\mathfrak{g})$ that enable one to write $U Y Z=W_{1} W_{2}$ for some $U, Y, Z, W_{1}, W_{2} \in \mathfrak{g}$ such that $W_{1} W_{2} v \neq 0$, then $F_{3} \neq 0$.

Theorem 4.1. Let $X \subset \mathbb{P} V$ be a homogeneous variety which is a product of irreducible varieties of type $G / P_{\alpha}$, with $G$ simple and $\alpha$ not short. Let $x \in X$. Then $X$ is generalized minuscule if and only if the only nonzero differential invariants of $X$ at $x$ are its fundamental forms. 
Remark 4.2. The result is false if one drops the hypothesis of $X$ being homogeneous, even if one requires $X$ to be smooth and $x$ to be a general point. However we believe it is true if one does not require $X$ to be homogenous, but requires that the only nonzero differential invariants are the fundamental forms at all points of $X$.

Remark 4.3. If one thinks of the generalized minuscule varieties as those admitting a Hermitian symmetric metric, then one could prove the result by observing that $\nabla I I^{\text {herm }}=0$, where $I I^{\text {herm }}$ is the Hermitian second fundamental form and $\nabla$ is the covariant differential operator. $I I$, the projective second fundamental form, is the holomorphic part of $I I^{\text {herm }}$ and $F_{3}$ is the holomorphic part of $\nabla I I^{\text {herm }}$, and so on. Since we will stay in the projective category, we will argue along different lines which will give more precise information about the invariants $F_{k}$ in the other cases.

Proof. By 四, the differential invariants of Segre products and Veronese re-embeddings can be computed from the original embeddings and the theorem holds in general if it holds for irreducible embeddings. Thus we restrict ourselves to the case where $X=G / P_{\alpha} \subset \mathbb{P} V$ is irreducible and in its minimal embedding.

Let $v \in V$ be a highest weight vector. Then $T_{[v]} X \simeq \mathfrak{g}_{+} v$, where

$$
\mathfrak{g}_{+}:=\bigoplus_{\left\{\beta \in \Delta \mid\left(\omega_{\alpha}, \beta\right)>0\right\}} \mathfrak{g}_{-\beta} .
$$

Here $\Delta$ denotes the set of all roots, $\omega_{\alpha}$ denotes the weight dual to the coroot of $\alpha$ and $($,$) the$ invariant bilinear form. Consider $X_{1} \cdots X_{k} v$, where $X_{j} \in \mathfrak{g}_{+}$. In order to have some nonzero component in $N_{p}$ with $p<k$, some bracket among the $X_{j}$ must be nonzero. However, if $V$ is minuscule, then $[X, Y]=0$ for all $X, Y \in \mathfrak{g}_{+}$. This shows that $F_{k+l, X}^{k}=0$ for all $l>0$ when $X$ is minuscule.

Conversely, let $Y=$ Base $\left|I I_{X,[v]}\right|$. By, e.g., [7], $T_{[v]} X$ splits as a sum $T=T_{1} \oplus T_{2} \oplus T_{3} \oplus \cdots$ of irreducible $H$-modules, where $H$ is a maximal semisimple subgroup of $P_{\alpha}$, and $T=T_{1}$ if and only if $\alpha$ is minuscule.

Let $Z v \in T_{1}$ be a general point of the cone over $\sigma(Y)$. We may write $Z=Z_{1}+Z_{2}$ with $Z_{j}^{2} v=0$, i.e., $Z_{j} v \in \hat{Y}$, the cone over $Y$. We calculate

$$
\begin{aligned}
Z^{2} v & =\left(Z_{1} Z_{2}+Z_{2} Z_{1}\right) v=\left(2 Z_{1} Z_{2}-\left[Z_{1}, Z_{2}\right]\right) v \\
Z^{3} v & =\left(2\left[Z_{1}, Z_{2}\right]\left(Z_{1}-Z_{2}\right)+\left(Z_{1}-Z_{2}\right)\left[Z_{1}, Z_{2}\right]\right) v .
\end{aligned}
$$

Note that if $Z^{3} v$ is not zero, it is in $N_{2}$ and thus $F_{3}(Z v, Z v, Z v) \neq 0$. We can write $Z^{3} v \equiv 3\left[Z_{1}, Z_{2}\right]\left(Z_{1}-Z_{2}\right) v \bmod T_{3}$; thus we are reduced to showing that for generic $Z$, $W_{2} W_{1} v \neq 0$ where $W_{2}=\left[Z_{1}, Z_{2}\right] \in T_{2}$ and $W_{1}=\left(Z_{1}-Z_{2}\right) \in T_{1}$.

If $X$ is not minuscule, i.e., if $\mathfrak{g}_{2} \simeq T_{2} \neq 0$, then $\mathfrak{g}_{2}=\left[\mathfrak{g}_{1}, \mathfrak{g}_{1}\right]$. In this case $\left[Z_{1}, Z_{2}\right] \neq 0$ because $Y \subset \mathbb{P} T_{1}$ is linearly nondegenerate and $Z_{1}, Z_{2}$ are general points of $\hat{Y}$. If $\alpha$ is not short, then $T_{1}^{*} \otimes T_{2}^{*} \subset|I I|$ by [7], 2.19, and thus $W_{2} W_{1} v \neq 0$ for all $W_{1} \in T_{1} \backslash 0, W_{2} \in T_{2} \backslash 0$. This proves our claim.

\section{Proof of the minuscule case}

Strategy. We need to show that the outputs of the minuscule algorithm are indeed minuscule varieties, and that all minuscule varieties arise by the algorithm.

In our algorithm, the rational map $\phi$ is defined such that at the point $x=[1,0, \ldots, 0] \in X$, the only nonzero differential invariants are the fundamental forms. Moreover, $\left|\mathbb{F F}_{X, x}^{k}\right|=$ 
$I_{k}\left(\sigma_{k-1}(Y)\right)$ and $Y$ is generalized minuscule. (Here and below, for an algebraic set $Z \subset \mathbb{P} V$, $I_{k}(Z) \subset S^{k} V^{*}$ denotes the component of the ideal of $Z$ in degree $k$.) By [7], 2.19 and 3.8, and Theorem 4.1, any potential minuscule variety must be constructed out of a generalized minuscule variety $Y$ by a mapping of the form $\phi$.

We need to show that the additional hypothesis that $\mathcal{T}(Y)$ is linearly nondegenerate is necessary and sufficient to imply that $X=G / P_{\alpha} \subset \mathbb{P V}$, where $G$ is simple and $\alpha$ is minuscule.

We proceed by constructing the Lie algebra $\mathfrak{g}$, showing that there is a unique candidate for $\mathfrak{g}$ and that this candidate can be given an appropriate Lie algebra structure if and only if $\mathcal{T}(Y)$ is linearly nondegenerate.

When such a $\mathfrak{g}$ exists, we then observe that the associated minuscule variety has the same projective differential invariants as $X$ at a point and therefore the two must coincide.

Analysis of a minuscule grading. A minuscule root of a simple Lie algebra $\mathfrak{g}$ induces a three step $\mathbb{Z}$-grading of $\mathfrak{g}$

$$
\mathfrak{g}=\mathfrak{g}_{-1} \oplus \mathfrak{g}_{0} \oplus \mathfrak{g}_{1},
$$

where $\mathfrak{g}_{0}=\mathfrak{h} \oplus \mathbb{C}$ is a reductive Lie algebra with one dimensional center and having semisimple part $\mathfrak{h}$. In addition $\mathfrak{g}_{1}$ can be identified with $T=T_{[v]} G / P_{\alpha}$, where $v \in V$ is a highest weight vector. The closed orbit $Y=H / Q \subset \mathbb{P} T$ is a generalized minuscule variety (see [12] and [7]).

This $\mathbb{Z}$-grading of $\mathfrak{g}$ induces $\mathfrak{g}_{0}$-module structures on $\mathfrak{g}_{ \pm 1}$ which determine the brackets $\left[\mathfrak{g}_{0}, \mathfrak{g}_{ \pm 1}\right]$. Moreover, $\left[\mathfrak{g}_{1}, \mathfrak{g}_{1}\right]=\left[\mathfrak{g}_{-1}, \mathfrak{g}_{-1}\right]=0$. Thus given $\mathfrak{g}_{ \pm 1}$ with their $\mathfrak{g}_{0}$-module structures, the only bracket we have not yet determined is $\left[\mathfrak{g}_{1}, \mathfrak{g}_{-1}\right]$. This bracket must be a $\mathfrak{g}_{0}$-equivariant map

$$
\mathfrak{g}_{-1} \otimes \mathfrak{g}_{1} \longrightarrow \mathfrak{g}_{0}=\mathfrak{h} \oplus \mathbb{C}
$$

This map has two components, the second of which induces a natural duality between $\mathfrak{g}_{1} \simeq T$ and $\mathfrak{g}_{-1} \simeq T^{*}$, hence an identification of $\mathfrak{g}_{-1}$ with $T^{*}$. We denote the first component by $\theta$.

Consider the Killing form $B_{\mathfrak{g}}$ of $\mathfrak{g}$. Up to some nonzero constants, we must have, for $X_{0}, Y_{0} \in \mathfrak{g}_{0}$ and $X_{ \pm 1} \in \mathfrak{g}_{ \pm 1}$,

$$
B_{\mathfrak{g}}\left(X_{0}, Y_{0}\right)=B_{\mathfrak{h}}\left(X_{0}, Y_{0}\right), \quad B_{\mathfrak{g}}\left(X_{1}, X_{-1}\right)=\left\langle X_{1}, X_{-1}\right\rangle,
$$

where this last bracket is given by the natural pairing between $\mathfrak{g}_{1}$ and $\mathfrak{g}_{-1}$. The invariance of the Killing form then implies that

$$
B_{\mathfrak{h}}\left(X_{0},\left[X_{1}, X_{-1}\right]\right)=B_{\mathfrak{g}}\left(X_{0},\left[X_{1}, X_{-1}\right]\right)=B_{\mathfrak{g}}\left(\left[X_{0}, X_{1}\right], X_{-1}\right)=\left\langle\left[X_{0}, X_{1}\right], X_{-1}\right\rangle .
$$

In particular, the map $\theta$ is determined, up to a constant, by the $\mathfrak{g}_{0}$ action on $\mathfrak{g}_{1}$ and its pairing with $\mathfrak{g}_{-1}$. The above identity can be rewritten, again up to a constant, for $u^{*} \in \mathfrak{g}_{-1}$, $v \in \mathfrak{g}_{1}$, as

$$
\theta\left(u^{*} \otimes v\right)=\sum_{i}\left\langle u^{*}, X_{i} v\right\rangle Y_{i},
$$

where $X_{i}$ and $Y_{i}$ are dual bases of $\mathfrak{h}$ with respect to its Killing form.

Construction of the Lie algebra. Now we are given a generalized minuscule variety $Y \subset \mathbb{P} T$ for a semisimple Lie algebra $\mathfrak{h}$, and we want to construct a new Lie algebra $\mathfrak{g}$ and a minuscule variety of $\mathfrak{g}$. In particular, $\mathfrak{g}$ must be endowed with a $\mathbb{Z}$-grading as above, and we have just seen that the only possible compatible Lie bracket in $\mathfrak{g}$ is determined, up to the multiplication of $\theta$ by some constant, by the $\mathfrak{h}$-module structure of $T$. It remains to see whether the constant can be chosen such that the Jacobi identities hold. 
Note that, assuming $\mathfrak{g}$ is a Lie algebra, it must be simple. Indeed, any nontrivial ideal $\mathfrak{i} \subseteq \mathfrak{g}$ is an $\mathfrak{h}$-module. If the center $\mathbb{C} \subset \mathfrak{g}_{0}$ were not in $\mathfrak{i}$, then neither would $T$ nor $T^{*}$ as $\mathbb{C} \subset\left[T, T^{*}\right]$. But since $[\mathfrak{h}, T]=T,\left[\mathfrak{h}, T^{*}\right]=T^{*}, \mathfrak{i}$ must be zero in this case. Now if $\mathbb{C} \subset \mathfrak{i}$ we see $T, T^{*} \subset \mathfrak{i}$ as well and thus $\mathfrak{h} \subset \mathfrak{i}$ so $\mathfrak{i}=\mathfrak{g}$.

Proposition 5.1. The bracket defined above endows $\mathfrak{g}$ with the structure of a Lie algebra for some constant multiple of $\theta$ if and only if $\mathcal{T}(Y) \subset \mathbb{P}\left(\wedge^{2} T\right)$ is linearly nondegenerate.

The choice of constant for $\theta$ is unique; see the proof of Lemma 5.2 below.

Proof. To determine if $\mathfrak{g}$ is actually a Lie algebra, we need to check the Jacobi identity. This identity can be split into a number of graded parts, which are of different natures. The $\left(\mathfrak{g}_{0}, \mathfrak{g}_{0}, \mathfrak{g}_{i}\right)$ identities follow because $\mathfrak{g}_{i}$ is a $\mathfrak{g}_{0}$-module, while the $\left(\mathfrak{g}_{0}, \mathfrak{g}_{i}, \mathfrak{g}_{j}\right)$ identities follow because the bracket $\left[\mathfrak{g}_{i}, \mathfrak{g}_{j}\right] \rightarrow \mathfrak{g}_{i+j}$ is $\mathfrak{g}_{0}$-equivariant. It remains to verify the $\left(\mathfrak{g}_{i}, \mathfrak{g}_{j}, \mathfrak{g}_{k}\right)$ identities for $i, j, k \neq 0$. By symmetry, the only identity to check is the one involving $v, w \in \mathfrak{g}_{1}=T$ and $u^{*} \in \mathfrak{g}_{-1}=T^{*}$. We must show that

$$
\left[\left[u^{*}, v\right], w\right]+\left[[v, w], u^{*}\right]+\left[\left[w, u^{*}\right], v\right]=\theta\left(u^{*} \otimes v\right) w-\theta\left(u^{*} \otimes w\right) v+\left\langle u^{*}, v\right\rangle w-\left\langle u^{*}, w\right\rangle v=0
$$

i.e., that

$$
t^{*}\left(\theta\left(u^{*} \otimes v\right) w-\theta\left(u^{*} \otimes w\right) v\right)=\left\langle t^{*}, v\right\rangle\left\langle u^{*}, w\right\rangle-\left\langle t^{*}, w\right\rangle\left\langle u^{*}, v\right\rangle
$$

for all $t^{*} \in T^{*}$. The map $\left(v, w, u^{*}, t^{*}\right) \mapsto\left\langle t^{*}, v\right\rangle\left\langle u^{*}, w\right\rangle-\left\langle t^{*}, w\right\rangle\left\langle u^{*}, v\right\rangle$, when considered as an element of $\Lambda^{2} T^{*} \otimes \Lambda^{2} T$, is just twice the identity, so we must prove that $\Theta \in \Lambda^{2} T^{*} \otimes \Lambda^{2} T$ defined by

$$
\Theta(v \wedge w)\left(u^{*}, t^{*}\right)=t^{*}\left(\theta\left(u^{*} \otimes v\right) . w-\theta\left(u^{*} \otimes w\right) \cdot v\right)
$$

is a homothety, since $\theta$ is only determined up to a constant multiple.

Lemma 5.2. $\Theta \mid\langle\mathcal{T}(Y)\rangle$ is a homothety.

Proof. Given a Cartan subalgebra $\mathfrak{t}$ of $\mathfrak{h}$ and a basis of the corresponding root system $\Delta$, we choose an adapted basis $E_{\beta}, H_{j}, E_{-\beta}$ of $\mathfrak{h}$, where $\beta \in \Delta_{+}$is a positive root, $B_{\mathfrak{h}}\left(E_{\beta}, E_{-\beta}\right)=1$, and the $H_{j}$ give an orthonormal basis of $\mathfrak{t}$ with respect to the Killing form. Then

$$
\Theta(v \wedge w)=\sum_{\beta \in \Delta_{+}}\left(E_{\beta} v \wedge E_{-\beta} w+E_{-\beta} v \wedge E_{\beta} w\right)+2 \sum_{j} H_{j} v \wedge H_{j} w .
$$

Now assume, to simplify notation, that $Y$ is a minuscule variety of the simple group $H$, so that $Y=H / Q_{\zeta}$ for some parabolic subgroup $Q_{\zeta}$ of $H$ corresponding to a minuscule root $\zeta$ of $\mathfrak{h}$. Then $T$ is the fundamental $\mathfrak{h}$-module of highest weight the fundamental weight $\omega$ dual to the coroot of $\zeta$. If $Y$ is a Segre product of minuscule varieties, the argument will be unchanged. If $Y$ is a Segre product of Veronese re-embeddings of minuscule varieties, it is necessary to keep track of the degrees of the re-embeddings in the argument.

In the computation above, we may suppose that $v \in Y$ and $w$ is tangent to a line of $Y$ passing through $v$ (as $\mathcal{T}(Y)$ being linearly nondegenerate implies $Y$ is linearly nondegenerate). Since $Y$ is $H$-homogeneous, we may assume that $v$ is a highest weight vector for $T$. In this case, when $\beta$ is a positive root, $E_{\beta} v=0$, and $E_{-\beta} v=0$ unless $\beta$ has coefficient one on the minuscule root $\zeta$. By linearity, we may assume that $w=E_{-\gamma} v$ for some positive root $\gamma$. Then, for $\beta$ as above, $E_{\beta} w=0$ unless $\beta=\gamma$, and thus

$$
\begin{aligned}
\Theta(v \wedge w v) & =E_{-\gamma} v \wedge E_{\gamma} w+2 \Sigma_{j} H_{j} v \wedge H_{j} w \\
& =w \wedge E_{\gamma} E_{-\gamma} v+2 \Sigma_{j} \omega\left(H_{j}\right)(\omega-\gamma)\left(H_{j}\right) v \wedge w \\
& =w \wedge\left[E_{\gamma}, E_{-\gamma}\right] v+2(\omega, \omega-\gamma) v \wedge w .
\end{aligned}
$$


Here we denoted by $($,$) the pairing on \mathfrak{t}^{*}$ dual to the Killing form. We have $\left[E_{\gamma}, E_{-\gamma}\right]=$ $\frac{(\gamma, \gamma)}{2} H_{\gamma}$, where $H_{\gamma}$ is the coroot of $\gamma$ (see [1]). Finally,

$$
\omega\left(\left[E_{\gamma}, E_{-\gamma}\right]\right)=\frac{(\gamma, \gamma)}{2} \omega\left(H_{\gamma}\right)=(\omega, \gamma)=(\omega, \zeta)
$$

because the Killing form identifies the coroot of $\gamma$ with $\frac{2 \gamma}{(\gamma, \gamma)}$ and the last equality holds because $\gamma$ has coefficient one on the simple root $\zeta$. The lemma is proved.

Remark 5.3. It turns out that to accommodate semisimple Lie algebras, it will be necessary to normalize long roots to have the same length independent of the Lie algebra. We choose length two, so one obtains $\left.\Theta\right|_{\langle\mathcal{T}(Y)\rangle}=2((\omega, \omega)-2) I d$.

For the opposite direction, we introduce a definition.

Definition 5.4. Let $\mathfrak{h}$ be a semisimple Lie algebra and let $U$ be an $\mathfrak{h}$-module. We will say $U$ is $C$-irreducible if the Casimir operator $C_{U}$ acts on $U$ as a homothety. In particular, every irreducible $\mathfrak{h}$-module is $C$-irreducible. If $U$ is $C$-irreducible, we let $c_{U}$ be the constant such that $C_{U}=c_{U} I d_{U}$.

We say that $U$ is almost $C$-irreducible if $U=\mathbb{C} \oplus V$ where $V$ is $C$-irreducible.

Lemma 5.5. Let $\mathfrak{h}$ be semisimple, let $T$ be an irreducible $\mathfrak{h}$-module and let $\Theta$ be defined as above. Then $\Theta=C_{\wedge^{2} T}-2 c_{T} I d_{\wedge^{2} T}$. In particular, $\Theta$ is a homothety if and only if $\Lambda^{2} T$ is C-irreducible.

Proof. Let $X_{i}, Y_{i}$ be bases of $\mathfrak{h}$ dual with respect to the Killing form. Let $v, w \in T$. Then

$$
\begin{aligned}
C_{\wedge^{2} T}(v \wedge w) & =\Sigma_{i} X_{i} Y_{i}(v \wedge w) \\
& =\Sigma_{i}\left(X_{i} Y_{i} v\right) \wedge w+\left(Y_{i} v\right) \wedge X_{i} w+\left(X_{i} v\right) \wedge Y_{i} w+v \wedge\left(X_{i} Y_{i} w\right) \\
& =2 c_{T} v \wedge w+\Theta(v \wedge w),
\end{aligned}
$$

which implies our claim.

Our proof of Proposition 5.1 will be complete once we have proven the following lemma:

Lemma 5.6. Let $T$ be a generalized minuscule module of a semisimple Lie algebra $\mathfrak{h}$. Let $Y \subset \mathbb{P} T$ be the corresponding generalized minuscule variety. If $\wedge^{2} T$ is $C$-irreducible, then $\mathcal{T}(Y)$ is linearly nondegenerate.

We first prove the case where $\mathfrak{h}$ is simple, which follows from the following lemma:

Lemma 5.7. Let $\mathfrak{h}$ be a simple Lie algebra and let $T$ be a minuscule $\mathfrak{h}$-module or a symmetric power of a minuscule $\mathfrak{h}$-module. Suppose that $\wedge^{2} T$ is $C$-irreducible. Then $\wedge^{2} T$ is irreducible.

Proof. Let $\omega=\omega_{\alpha}$ be the highest weight of the minuscule module $T$. The highest weight of $\wedge^{2} T$ is $2 \omega-\alpha$. Suppose that $\wedge^{2} T$ has an irreducible component of another highest weight. This weight $\mu$ must be the sum of two distinct weights of $T$. Hence, since $T$ is minuscule, $\mu=u \omega+v \omega$ for two distinct elements $u, v$ of the Weyl group. We show that $c_{\mu}<c_{2 \omega-\alpha}$, which will prove that $\wedge^{2} T$ is not $C$-irreducible. Since $(\alpha, 2 \rho-\alpha)=0$, we have

$$
c_{2 \omega-\alpha}-c_{\mu}=2(\omega, \omega)-2(u \omega, v \omega)+(2 \omega-u \omega-v \omega, 2 \rho) .
$$


Write $u \omega=\omega-\sum_{\gamma} n_{\gamma}(u) \gamma$, where the sum is over the simple roots. The coefficients $n_{\gamma}(u)$ are non-negative, and $n_{\alpha}(u)>0$ when $u \omega \neq \omega$. Thus

$$
\begin{aligned}
(u \omega, v \omega) & =\left(\omega, u^{-1} v \omega\right)=\left(\omega, \omega-\sum_{\gamma} n_{\gamma}\left(u^{-1} v\right) \gamma\right)=(\omega, \omega)-n_{\alpha}\left(u^{-1} v\right) \\
(u \omega, 2 \rho) & =\left(\omega-\sum_{\gamma} n_{\gamma}(u) \gamma, 2 \rho\right)=(\omega, 2 \rho)-\sum_{\gamma}(\gamma, \gamma) n_{\gamma}(u) .
\end{aligned}
$$

This implies that

$$
c_{2 \omega-\alpha}-c_{\mu}=\sum_{\gamma}(\gamma, \gamma)\left(n_{\gamma}(u)+n_{\gamma}(v)\right)+2 n_{\alpha}\left(u^{-1} v\right)-4 .
$$

This number is positive: this is clear if $u \omega, v \omega \neq \omega$, since $n_{\alpha}(u), n_{\alpha}(v), n_{\alpha}\left(u^{-1} v\right)$ are then all positive; otherwise, we may suppose that $u=i d$, in which case

$$
\left.c_{2 \omega-\alpha}-c_{\lambda}=\sum_{\gamma \neq \alpha}(\gamma, \gamma) n_{\gamma}(v)\right)+4 n_{\alpha}(v)-4,
$$

with $n_{\alpha}(v)>0$, and this number is clearly positive unless $v \omega=\omega-\alpha$, which is excluded.

The case of symmetric powers of a minuscule module is similar.

We consider now the case where $\mathfrak{h}$ is not simple, and $T=T_{1} \otimes \cdots \otimes T_{r}$, where $T_{i}$ is a $\mathfrak{h}_{i}$-module of the form $S^{d_{i}} U_{i}$, with $U_{i}$ fundamental and minuscule. For $r=3$, we have

$$
\wedge^{2} T=\wedge^{2} T_{1} \otimes S^{2} T_{2} \otimes S^{2} T_{3} \oplus S^{2} T_{1} \otimes \wedge^{2} T_{2} \otimes S^{2} T_{3} \oplus S^{2} T_{1} \otimes S^{2} T_{2} \otimes \wedge^{2} T_{3} \oplus \wedge^{2} T_{1} \otimes \wedge^{2} T_{2} \otimes \wedge^{2} T_{3} .
$$

For such a module to be $C$-irreducible, we first need that each $S^{2} T_{i}$ and $\wedge^{2} T_{i}$ be $C$-irreducible. But we always have $c_{\wedge^{2} T_{i}}<c_{S^{2} T_{i}}$, hence a contradiction. For the same reasons we cannot have $r \geq 4$.

If $r=2$, then $\wedge^{2} T=\wedge^{2} T_{1} \otimes S^{2} T_{2} \oplus S^{2} T_{1} \otimes \wedge^{2} T_{2}$ is $C$-irreducible if and only if the $S^{2} T_{i}$ and $\wedge^{2} T_{i}$ are $C$-irreducible. To see this, recall that for a representation $V_{\omega}$, if $S^{2} V$ and $\Lambda^{2} V$ are $C$-irreducible, then with the normalization of long roots having length two, $c_{S^{2} V}=c_{\Lambda^{2} V}+4$, so $c_{\Lambda^{2} T_{1} \otimes S^{2} T_{2}}=c_{S^{2} T_{1}}-4+c_{S^{2} T_{2}}=c_{S^{2} T_{1} \otimes \Lambda^{2} T_{2}}$. By the above lemma, this implies that $\wedge^{2} T_{i}$ and $S^{2} T_{i}$ are irreducible. But then $\mathcal{T}(Y)$ is linearly nondegenerate.

We record the following lemma, which we will need in $\S 6$.

Lemma 5.8. If $\wedge^{2} T$ is almost $C$-irreducible, then $\langle\mathcal{T}(Y)\rangle$ has codimension one.

Proof. The lemma follows from Lemma 5.7 when $\mathfrak{h}$ is simple. For $T=T_{1} \otimes \cdots \otimes T_{r}$, we check as above that $r \geq 3$ is not possible unless $r=3$ and the $T_{i}$ are two-dimensional. Finally, for $r=2$, we can suppose that $S^{2} T_{1}$ and $\wedge^{2} T_{2}$ are $C$-irreducible, while $\wedge^{2} T_{1}=\mathbb{C} \oplus A_{1}$ and $S^{2} T_{2}=\mathbb{C} \oplus A_{2}$ are $C$-irreducible. But then $\wedge^{2} T$ contains $A_{1}$ and $A_{1} \otimes A_{2}$, and the Casimir operator must act on both modules as the same constant. This leads to a contradiction unless $A_{1}$ is zero, which means that $T_{1}=\mathbb{C}^{2}$ is two-dimensional, and $\wedge^{2} T=S^{2} T_{2} \oplus S^{2} \mathbb{C}^{2} \otimes \wedge^{2} T_{2}$. We note that in this case $\langle\mathcal{T}(Y)\rangle$ must have codimension one.

Remark 5.9. The minuscule and adjoint algorithms can be generalized to construct all $G / P$ 's, however the resulting rational maps get more and more complicated. The corresponding construction of a $\mathbb{Z}$-graded Lie algebra $\mathfrak{g}$ from a Lie algebra $\mathfrak{h}$ equipped with a representation $T_{1}$ (by gluing in a node to the marked Dynkin diagram) is easier to describe and even provides an effective algorithm for producing $\mathbb{Z}$-graded affine Lie algebras. 
One takes $\mathfrak{g}_{0}=\mathfrak{h}+\mathbb{C}$ and $\mathfrak{g}_{1}=T_{1}$ as before. Next, for $\mathfrak{g}_{2}$, one takes the $\mathfrak{h}$-module complementary to $\langle\mathcal{T}(Y)\rangle$ in $\Lambda^{2} T_{1}$, and denote the corresponding closed $H$-orbit $Y_{2} \subset \mathbb{P a}_{2}$. Now there are further Jacobi identities to check, but these can be checked geometrically by considering the linear span of the natural incidence variety $Y_{12} \subset Y \times Y_{2} \subset \mathbb{P} S_{21}\left(T_{1}\right)$. If it is linearly full, one has already constructed $\mathfrak{g}$. If not, one takes the complement of its linear span as $\mathfrak{g}_{3}$. At each step one needs only to consider the linear span of the analogously defined $Y_{1 k}$. (This is because N. Mok has observed that in a $\mathbb{Z}$-graded Lie algebra generated by $\mathfrak{g}_{1}$, one always has $\left[\mathfrak{g}_{p}, \mathfrak{g}_{q}\right] \subseteq\left[\mathfrak{g}_{1}, \mathfrak{g}_{p+q-1}\right]$ (personal communication).) By way of example, consider the $\mathbb{Z}$-gradings of $\mathfrak{e}_{8}{ }^{(1)}\left(\mathbb{Z}_{m}\right.$-gradings of $\left.\mathfrak{e}_{8}\right)$ obtained from the extremal nodes: from $\alpha_{1}$ we have a $\mathbb{Z}_{2}$-grading with $\mathfrak{g}_{0}=\mathfrak{d}_{8}+\mathbb{C}, \mathfrak{g}_{1}=V_{\omega_{8}}$ (spin representation), $\alpha_{2}$ yields a $\mathbb{Z}_{3}$-grading with $\mathfrak{g}_{0}=\mathfrak{a}_{8}+\mathbb{C}, \mathfrak{g}_{1}=V_{\omega_{3}}, \mathfrak{g}_{2}=V_{\omega_{6}}$, and $\alpha_{8}$ yields a $\mathbb{Z}_{2}$-grading with $\mathfrak{g}_{0}=\mathfrak{e}_{7}+\mathfrak{a}_{1}+\mathbb{C}$, $\mathfrak{g}_{1}=V_{\omega_{7}}^{\mathfrak{e}_{7}} \otimes V_{\omega_{1}}^{\mathfrak{a}_{1}}$

One could attempt to continue the algorithm for general Kac-Moody Lie algebras, but since there will be exponential growth of the factors, the algorithm is not at all effective. Note that for affine Lie algebras the algorithm is considerably more explicit than the standard method of construction by taking the free Lie algebra and then quotienting out by the relations.

\subsection{Minuscule algorithm: the algebraic version}

We may rephrase the minuscule algorithm algebraically: Let $\mathfrak{h}=\mathfrak{h}_{1} \oplus \cdots \oplus \mathfrak{h}_{r}$ where the $\mathfrak{h}_{j}$ are constructed in an earlier run through the algorithm. Let $T_{j}$ be the corresponding representations constructed or a symmetric power of such representations, and let $T=$ $T_{1} \otimes \cdots \otimes T_{r}$. Let $\tilde{Y} \subset \mathbb{P} T^{*}$ be the closed $H$-orbit, let $V_{0}=\mathbb{C}, V_{1}=T, V_{2}=I_{2}(\tilde{Y}) \subseteq S^{2} T$, and let

$$
V_{k}=\left(V_{k-1} \otimes T\right) \cap S^{k} T,
$$

the prolongation of $V_{k-1}$ (see [7]).

Call $(\mathfrak{h}, T)$ admissible if $\langle\mathcal{T}(Y)\rangle=\mathbb{P} \Lambda^{2} T^{*}$. If $(\mathfrak{h}, T)$ is admissible, then

$$
\begin{aligned}
\mathfrak{g} & :=T^{*} \oplus(\mathfrak{h} \oplus \mathbb{C}) \oplus T, \\
V & :=\oplus_{j=0}^{\infty} V_{j}
\end{aligned}
$$

are respectively a simple Lie algebra, and a minuscule $\mathfrak{g}$-module. (The last sum is finite because the terms correspond to the generators of the ideals of successive secant varieties.)

Moreover, starting with $(\mathfrak{h}, T)=(0, \mathbb{C})$ and iterating the algorithm, one arrives at all pairs $(\mathfrak{g}, V)$, with $\mathfrak{g}$ simple and $V$ a minuscule $\mathfrak{g}$-module.

Remark 5.10. Instead of constructing $\mathfrak{g}$ first, one could first construct $V$ as above, define an action of each component of $\mathfrak{g}$ on $V$, and define the bracket in $\mathfrak{g}$ via the commutator of the actions on $\mathfrak{g}$. The algebra $\mathfrak{h}$ acts naturally on each $V_{j}$, one makes $\mathbb{C} \subset \mathfrak{g}_{0}$ act on each factor by a scalar, and $T^{*}$ acts naturally as a lowering (or "annihilation") operator $V_{j} \rightarrow V_{j-1}$ by differentiation. One makes $T$ act as a raising (or "creation") operator as follows: for $p \in V_{l-1}$, one defines $w . p$, up to a constant, as the projection of $w \circ p$ on $V_{l}$ (one must check that this is well defined).

For example, consider the case $Y=G(2, W), X=\mathbb{S}$. Here $V_{k}=\Lambda^{2(k+2)} W$ and the raising and lowering action corresponds to Clifford multiplication. Thus we see that minuscule representations define algebraic structures that are cousins of Clifford algebras.

Taking $V_{2}$ to be the ideal of a variety ensures the sum is finite. To study infinite dimensional representations, take $V_{2} \subseteq S^{2} T$ to be an h-invariant subspace whose successive prolongations do not terminate, e.g., one could take $V_{2}$ to be involutive (see [2] for the definition of involutivity). 


\section{Proofs of the adjoint theorems}

\subsection{The fundamental case}

Strategy. We need to prove that the adjoint algorithm is well defined, i.e., that $\tau(Y)$ is a quartic hypersurface, that the adjoint algorithm constructs all fundamental adjoint varieties, and that the adjoint algorithm only constructs fundamental adjoint varieties.

We begin, as in the minuscule algorithm, by studying the five step $\mathbb{Z}$-grading of a simple

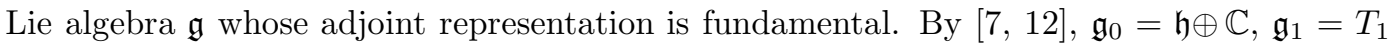
is a minuscule $\mathfrak{h}$-module and $\mathfrak{g}_{2}=\mathbb{C}$. Letting $Y_{1} \subset \mathbb{P} T_{1}$ denote the closed $H$-orbit, we show that a necessary and sufficient condition that the pair $\left(\mathfrak{h}, T_{1}\right)$ produces a simple Lie algebra $\mathfrak{g}$ together with its adjoint representation is that the linear span of $\mathcal{T}(Y)$ has codimension one. (Note that the line in $\Lambda^{2} T_{1}$, generated by the symplectic form of $T_{1}$, already implies that the complement of $\langle\mathcal{T}(Y)\rangle$ must contain a trivial $\mathfrak{h}$-module.)

We then determine the differential invariants of a fundamental adjoint variety, in particular, we show that $\tau(Y)$ must be a quartic hypersurface. We finally observe that the invariants agree with the differential invariants of the varieties $X$ constructed by the algorithm at our preferred point $[1,0, \ldots, 0] \in X$.

Analysis of the adjoint grading. Let $\mathfrak{g}$ be a simple Lie algebra whose adjoint representation is fundamental. We choose a Cartan subalgebra of $\mathfrak{g}$ and a basis of the corresponding root system $\Delta$. We denote the highest root by $\tilde{\alpha}$, and consider the corresponding five step grading $([2])$

$$
\mathfrak{g}=\mathfrak{g}_{-2} \oplus \mathfrak{g}_{-1} \oplus \mathfrak{g}_{0} \oplus \mathfrak{g}_{1} \oplus \mathfrak{g}_{2} .
$$

Here $\mathfrak{g}_{0}=\mathfrak{h} \oplus \mathbb{C}, \mathfrak{g}_{2}=\mathbb{C}$, and $\mathfrak{g}_{1}=T_{1}$ is a generalized minuscule $\mathfrak{h}$-module. $T_{1}$ is the contact hyperplane inside the tangent space to a point $x \in G / P_{\tilde{\alpha}} \subset \mathbb{P} \mathfrak{g}$ such that $x=\mathbb{P} \mathfrak{g}_{-2}$. Let $Y \subset \mathbb{P} T_{1}$ be the closed $H$-orbit, then $Y=$ Base $\left|I I_{G / P_{\tilde{\alpha}}, x}\right|$. (See [7].)

The Lie algebra structure of $\mathfrak{g}$ can be recovered as follows: the Killing form of $\mathfrak{g}$ induces an identification between $\mathfrak{g}_{-1}$ and $T_{1}^{*}$, and also between $\mathfrak{g}_{-2}$ and $\mathfrak{g}_{2}^{*}$. The bracket $\left[\mathfrak{g}_{1}, \mathfrak{g}_{1}\right] \rightarrow$ $\mathfrak{g}_{2}=\mathbb{C}$ defines a symplectic form on $T_{1}$. We choose $\omega^{*} \in \mathfrak{g}_{-2} \subset \Lambda^{2} T_{1}$ and $\omega \in \mathfrak{g}_{2} \subset \Lambda^{2} T_{1}$ dual with respect to the Killing form. By contraction, $\omega$ induces an identification of $T_{1}$ with $T_{1}^{*}$, and we let $u^{*}=\omega(u,$.$) . Then$

$$
\begin{array}{rlrl}
{[u, v]} & =p \omega^{*}(u, v) \omega & \\
{\left[u^{*}, v^{*}\right]} & =p^{*} \omega^{*}(u, v) \omega^{*} \quad \forall u, v \in T_{1}, \\
{\left[u^{*}, \omega\right]} & =m u, & & \\
{\left[u, \omega^{*}\right]} & =m^{*} u^{*} & \forall u \in T_{1},
\end{array}
$$

for some constants $p, p^{*}, m, m^{*} \in \mathbb{C}$. By the invariance of the Killing form,

$$
p \omega^{*}(u, v)=B_{\mathfrak{g}}\left([u, v], \omega^{*}\right)=B_{\mathfrak{g}}\left(u,\left[v, \omega^{*}\right]\right)=m^{*}\left\langle u, v^{*}\right\rangle=m^{*} \omega^{*}(u, v),
$$

hence $p=m^{*}$ and similarly, $p^{*}=m$.

There exist $l, o \in \mathbb{C}$ and an element $\mathbf{1}$ in the center of $\mathfrak{g}_{0}$ such that

$$
\begin{aligned}
{\left[u^{*}, v\right] } & =\omega^{*}(u, v) \mathbf{1}+l \theta\left(u^{*} \otimes v\right) \quad \forall u, v \in T, \\
{\left[\omega^{*}, \omega\right] } & =o \mathbf{1} .
\end{aligned}
$$

Note that 1 must act on $T_{1}$ by some constant $a$. The map $\theta$ must be symmetric in the sense that

$$
\theta\left(u^{*} \otimes v\right)=\theta\left(v^{*} \otimes u\right) \quad \forall u, v \in T_{1}
$$


because $\mathfrak{h}$ preserves the form $\omega$. The Jacobi identities give additional constraints on the scaling constants $l, m, m^{*}, o, a$, and one can check that $o=a=m=-m^{*}=2$. Moreover, we will see that $l$ is determined by the Jacobi identity. Thus the Lie algebra structure of $\mathfrak{g}$ is determined by the $\mathfrak{h}$-module structure of $T_{1}$.

Construction of the Lie algebra. Now we begin with an $\mathfrak{h}$-module $T_{1}$ and an $\mathfrak{h}$-invariant two-form $\omega$ on $T_{1}$ (defined up to scale) and attempt to define a Lie algebra structure on the vector space $\mathfrak{g}$ with its five step gradation as above. The bracket on $\mathfrak{g}$ is determined up to the constant $l$ and we must check if the Jacobi identities hold.

Proposition 6.1. Let $\mathfrak{h}$ be semisimple and let $T_{1}$ be a generalized minuscule $\mathfrak{h}$-module. Then there exists a choice of $l$ such that $\mathfrak{g}$, with the bracket defined above, is a Lie algebra if and only if $\operatorname{codim}\langle\mathcal{T}(Y)\rangle=1$. In this case, $\mathfrak{g}$ is simple and its adjoint representation is fundamental.

Proof. By the same argument as in the minuscule case, the only identity we need to verify is the $\left(\mathfrak{g}_{1}, \mathfrak{g}_{1}, \mathfrak{g}_{-1}\right)$ identity, that is, for all $u, v, w \in T_{1}$,

$$
l\left[\theta\left(w^{*} \otimes v\right) u-\theta\left(w^{*} \otimes u\right) v\right]=\omega^{*}(v, w) u-\omega^{*}(u, w) v-2 \omega^{*}(u, v) w .
$$

As before, we interpret this equation as an equality between endomorphisms of $\Lambda^{2} T_{1}$. The left hand side represents the action of the Casimir operator (plus some multiple of the identity), the first two terms of the right hand side represent twice the identity of $\Lambda^{2} T_{1}$, and the last term is the projection onto $\mathbb{C} \omega \subset \Lambda^{2} T_{1}$. Thus the identity above will hold, with an appropriate choice of $l$ if and only if $\Lambda^{2} T_{1}$ is almost $C$-irreducible. By Lemmas 5.2 and 5.7, this is true if and only if $\operatorname{codim}\langle\mathcal{T}(Y)\rangle=1$.

One sees that $\mathfrak{g}$ is simple by an argument similar to the minuscule case.

The adjoint variety. We now calculate the differential invariants of a fundamental adjoint variety. We consider the adjoint variety $G / P_{\tilde{\alpha}} \subset \mathbb{P} \mathfrak{g}$ as the orbit of $[\omega]$, and identify the tangent space of $G / P_{\tilde{\alpha}}$ at $[\omega]$ with $\mathbb{C} \oplus T_{1} \subset \mathfrak{g}_{0} \oplus \mathfrak{g}_{1}$. Let $X=x \omega^{*}+u^{*}$ denote a general element of $\mathfrak{g}_{-2} \oplus \mathfrak{g}_{-1}$, which implies $X \omega=2 x \mathbf{1}+2 u$ is a general element of $T_{[\omega]} G / P_{\tilde{\alpha}}$ and $G / P_{\tilde{\alpha}}$ is the projectivization of the closure of the union of elements of the form $\exp (X) \omega$. We compute

$$
\begin{aligned}
X \omega & =2 x \mathbf{1}+2 u, \\
X^{2} \omega & =8 x^{2} \omega^{*}+2 l \theta\left(u^{*} \otimes u\right), \\
X^{3} \omega & =-2 l \theta\left(u^{*} \otimes u\right) u^{*}, \\
X^{4} \omega & =2 l \omega^{*}\left(u, \theta\left(u^{*} \otimes u\right) u\right), \\
X^{5} \omega & =0 .
\end{aligned}
$$

This computation already shows that our map $\phi$ in the adjoint algorithm must indeed be of degree (at most) four. It remains to interpret the above equations. From the second line and the fact that by $[7]$, Base $|I I|=Y$, we obtain:

Proposition 6.2. $Y=$ Base $|I I| \subset \mathbb{P} T_{1}$ is as follows:

$$
\begin{aligned}
Y & =\mathbb{P}\left\{u \in T_{1} \mid \theta\left(u^{*} \otimes u\right)=0\right\} \\
& =\mathbb{P}\left\{u \in T_{1} \mid \omega^{*}(u, Z u)=0 \forall Z \in \mathfrak{h}\right\} .
\end{aligned}
$$

In particular, the second description implies that $u$ is $\omega^{*}$-orthogonal to the tangent space of its $H$-orbit. 
Remark 6.3. In general, the space of quadrics vanishing on a closed orbit $Y$ is the kernel of the natural map from $S^{2} T_{1}^{*}$ to $S^{(2)} T_{1}^{*}$, where $S^{(2)} T_{1}^{*}$ denotes the Cartan product of $T_{1}^{*}$ with itself. An important consequence of Proposition 6.2 is that

$$
S^{2} T_{1}=S^{(2)} T_{1} \oplus \mathfrak{h} .
$$

Finally, we prove that the terms of degree three and four above are the same as those in the adjoint algorithm:

Proposition 6.4. The tangential variety $\tau(Y) \subset \mathbb{P} T_{1}$ is the quartic hypersurface defined by the equation

$$
p(w)=\omega^{*}\left(\theta\left(w^{*} \otimes w\right) w, w\right)=B_{\mathfrak{h}}\left(\theta\left(w^{*} \otimes w\right), \theta\left(w^{*} \otimes w\right)\right)=0 .
$$

In particular, the singular locus of $\tau(Y)$ is given by the equations $\theta\left(w^{*} \otimes w\right) w=0$.

Proof. $\tau(Y)$ is nondegenerate because $I I I_{Y} \neq 0,([5), 10.1)$, hence it is a hypersurface.

We check that $w=v+X v \in \tau(Y)$ implies $p(w)=0$. Introduce the notation $\theta^{*}(u v)=$ $\theta^{*}(u \cdot v)=\theta\left(u^{*} \otimes v\right)$ and note that $\theta^{*}(u v)=\theta^{*}(v u)$. For all $v \in Y$, we have $\theta^{*}\left(v^{2}\right)=0$. Differentiating, we obtain $\theta^{*}(v \cdot X v)=0$ and $\theta^{*}\left((X v)^{2}\right)+\theta^{*}\left(v \cdot X^{2} v\right)=0$ for all $X \in \mathfrak{h}$. Thus $\theta^{*}\left(w^{2}\right)=\theta^{*}\left((X v)^{2}\right)$.

Now recall the identity

$$
l\left[\theta^{*}(r s) t-\theta^{*}(r t) s\right]=\omega^{*}(r, s) t-\omega^{*}(r, t) s+2 \omega^{*}(s, t) r,
$$

which was a consequence of the Jacobi identity on $\mathfrak{g}$ (see the proof of Proposition 6.1). Setting $r=s=X v$ and $t=v$, we obtain $l \theta^{*}\left((X v)^{2}\right) v=l \theta^{*}(v \cdot X v) v+\omega^{*}(X v, v) X v=0$. Similarly, setting $r=v, s=X^{2} v$, and $t=X v$, we obtain $l \theta^{*}\left(v \cdot X^{2} v\right) X v=2 \omega^{*}\left(X^{2} v, X v\right) v$. This implies that $\theta^{*}\left(w^{2}\right) w=-2 \omega^{*}\left(X^{2} v, X v\right) v$, so that finally

$$
p(w)=\omega^{*}\left(\theta^{*}\left(w^{2}\right) w, w\right)=-2 \omega^{*}\left(X^{2} v, X v\right) \omega^{*}(v, X v)=0 .
$$

$p$ must be irreducible as there is no invariant linear or quadratic form on $T$, so $p$ must be the equation of $\tau(Y)$.

Remark 6.5. It turns out that the smooth part of the tangential variety is a single $\mathfrak{g}$-orbit. We have no a priori proof of this observation. Such a proof would make the above computation unnecessary. A consequence of the observation is that the invariant ring of $T$ is free, and generated by the quartic polynomial $p$.

\subsection{Another perspective on the adjoint algorithm}

We may think of $\mathfrak{g}_{-2} \oplus \mathbb{C} \oplus \mathfrak{g}_{2} \subset \mathfrak{g}$ as a copy of $\mathfrak{s l}_{2}$, where $\mathbb{C}$ is the center of $\mathfrak{g}_{0}$. Thus we may write $\mathfrak{g}=T_{1} \oplus\left(\mathfrak{h} \oplus \mathfrak{s l}_{2}\right) \oplus T_{1}^{*}$ and think of our modified construction as a "quantization" (in the sense that $\mathbb{C} \rightarrow \mathfrak{s l}_{2}$ ) of the construction in the minuscule case.

If we let $\mathbb{C}^{2}$ be the standard $\mathfrak{s l}_{2}$ module, we can write $\mathfrak{g}$ more concisely (recalling that $T_{1}$ is a symplectic module, hence can be identified with its dual) as $\mathfrak{g}=\left(\mathfrak{h} \oplus \mathfrak{s l}_{2}\right) \oplus \mathbb{C}^{2} \otimes T_{1}$. All our constructions can be made using invariant tensors on these two spaces. In this way we recover the $\mathbb{Z}_{2}$-gradings of a simple Lie algebra obtained by marking the node(s) of the Dynkin diagram of the affine Lie algebra $\mathfrak{g}^{(1)}$ adjacent to $\tilde{\alpha}$. Here $\mathfrak{g}_{0}=\left(\mathfrak{h}+\mathfrak{s l}_{2}\right)$, and $\mathfrak{g}_{1}=\mathbb{C}^{2} \otimes T_{1}$. In particular, Proposition 6.1 can be (partly) rephrased as follows: 
Proposition 6.6. Let $\mathfrak{h}$ be a simple Lie algebra and $T$ an irreducible symplectic $\mathfrak{h}$-module, such that $\Lambda^{2} T$ is the sum of an irreducible module and a line. Then

$$
\mathfrak{g}=\left(\mathfrak{h} \oplus \mathfrak{s l}_{2}\right) \oplus \mathbb{C}^{2} \otimes T
$$

has a natural structure of simple Lie algebra.

One obtains the following models of the exceptional complex Lie algebras, where we denote by $V_{\lambda}$ the irreducible $\mathfrak{h}$-module of highest weight $\lambda$, and we follow the conventions of [1] for the fundamental weights:

$\begin{array}{ccc}\mathfrak{h} & T & \mathfrak{g} \\ \mathfrak{s l}_{2} & V_{3 \omega_{1}} & \mathfrak{g}_{2} \\ \mathfrak{s p}_{6} & V_{\omega_{3}} & \mathfrak{f}_{4} \\ \mathfrak{s l}_{6} & V_{\omega_{3}} & \mathfrak{e}_{6} \\ \mathfrak{s o}_{12} & V_{\omega_{6}} & \mathfrak{e}_{7} \\ \mathfrak{e}_{7} & V_{\omega_{1}} & \mathfrak{e}_{8} .\end{array}$

\subsection{The non-fundamental case}

To complete our proof of the classification of complex simple Lie algebras, we prove that the only simple Lie algebras for which the adjoint representation is not fundamental are $\mathfrak{s l}_{n}$ and $\mathfrak{s p}_{2 m}$.

Consider again the adjoint grading $\mathfrak{g}=\mathfrak{g}_{-2} \oplus \mathfrak{g}_{-1} \oplus \mathfrak{g}_{0} \oplus \mathfrak{g}_{1} \oplus \mathfrak{g}_{2}$ of the Lie algebra $\mathfrak{g}$. By [7], Proposition 4.2, $\mathfrak{g}_{1}$ has at most two irreducible components as an $\mathfrak{h}$-module, where $\mathfrak{h} \subset \mathfrak{g}_{0}$ is the semisimple part of $\mathfrak{g}_{0}$, and the smallest weights of these $\mathfrak{h}$-modules are the simple roots $\alpha_{i}$ such that $\tilde{\alpha}-\alpha_{i}$ is still a root.

First case. Suppose that $\mathfrak{g}_{1}$ is irreducible. If the adjoint representation is not fundamental, $\tilde{\alpha}$ is a multiple of some fundamental weight $\omega$ and thus $X=v_{d}(Z)$ where $Z \subset \mathbb{P} V_{\omega}$ is the closed orbit and $d>1$.

We give two proofs of this case:

First proof: Base $|I I|=\emptyset$ because a Veronese re-embedding of any variety contains no lines and a Veronese re-embedding of a homogeneous variety is such that its ideal is generated in degree two. However, we know that the quadratic equations of Base $|I I|$ in $S^{2} T_{1}^{*}$ are given by $\mathfrak{h}$. Thus $\mathfrak{h}=S^{(2)} T_{1}^{*}$, since the other components of $S^{2} T_{1}^{*}$ all vanish on the closed orbit of $\mathbb{P} T_{1}$. So $\mathfrak{h}$ itself is not fundamental, and by induction on the dimension we conclude that $\mathfrak{h}$ must be a symplectic Lie algebra and $T_{1}$ is its natural representation.

Second proof: By [⿶] 3.10, $|I I|=S^{2} T_{1}^{*}$. Since $N_{2} \simeq \mathfrak{h} \oplus \mathfrak{g}_{1} \oplus \mathfrak{g}_{2}$, we see $|I I|=\mathfrak{h}$ and in particular that $\mathfrak{h} \simeq S^{2} T_{1}^{*}$, i.e, that $\mathfrak{h}=\mathfrak{s} p_{2 m}$ for some $m$ and $T_{1}$ is its standard representation. Since the $\mathfrak{h}$-module structure of $T_{1}$ completely determines $\mathfrak{g}$, this implies that $\mathfrak{g}$ is also a symplectic Lie algebra.

In this case our construction works as well, only it is degenerate, of degree two. Taking $Y=\emptyset$, the rational map $\mathbb{P}^{n} \rightarrow \mathbb{P}^{N}$ is by the quadrics vanishing on $Y$, that is, all quadrics. If one adopts the convention that $\tau(Y)=\tau(Y)_{\text {sing }}=\mathbb{P} T_{1}$, then this construction becomes consistent with the adjoint algorithm.

Second case. Suppose that $\mathfrak{g}_{1}$ is reducible, hence the sum of two dual $\mathfrak{g}$-modules $U$ and $U^{*}$ (this duality comes from the bracket $\left[\mathfrak{g}_{1}, \mathfrak{g}_{1}\right] \rightarrow \mathfrak{g}_{2}$ ).

Lemma 6.7. $\mathbb{P} U$ is $H$-homogeneous. 
Proof. Let the simple root $\alpha$ be the smallest weight of $U$. Then the highest root $\tilde{\alpha}$ has coefficient one on $\alpha$, and the weights $\gamma$ of $U$ are the other positive roots with this property. We write $\gamma=\sum_{\beta \text { simple }} n_{\beta}(\gamma) \beta$. Note that $n(\alpha, \tilde{\alpha})=1$ so that

$$
n(\tilde{\alpha}, \alpha)=2+\sum_{\beta \neq \alpha} n_{\beta}(\tilde{\alpha}) n(\beta, \alpha)>0 .
$$

The coefficients $n(\beta, \alpha)$ are the Cartan numbers, which are negative for $\beta \neq \alpha$. Now $n_{\beta}(\gamma) \leq$ $n_{\beta}(\tilde{\alpha})$ for any positive root $\gamma$ and any simple $\operatorname{root} \beta$ so the equation above implies that $n(\tilde{\alpha}, \gamma)>0$ for each root $\gamma$ that is a weight of $U$. Therefore, $\gamma-k \alpha$ is a root for some $k>0$ and in particular $\gamma-\alpha$ is a root.

Geometrically, letting $Y$ denote the smallest $H$-orbit in $\mathbb{P} U$ and $v_{\alpha} \in U$ a vector of weight $\alpha$, this implies that $\hat{T}_{\left[v_{\alpha}\right]} Y=\mathfrak{h} v_{\alpha}=U$, i.e., that $Y=\mathbb{P} U$.

Let $v=u+u^{*} \in \mathfrak{g}_{1}=U \oplus U^{*}$. Now $v \in$ Base $|I I|$ if and only $\omega^{*}(v, X v)=2\left\langle u, X u^{*}\right\rangle=0$ for every $X \in \mathfrak{h}$. By the lemma, this is the case if and only if $u$ or $u^{*}$ is zero, i.e.,

$$
\text { Base }|I I|=\mathbb{P} U \sqcup \mathbb{P} U^{*} \subset \mathbb{P}\left(U \oplus U^{*}\right) \text {. }
$$

Moreover, $I_{2}$ (Base $\left.|I I|\right)=U \otimes U^{*} \subset S^{2} \mathfrak{g}_{1}^{*}=S^{2} U \oplus U \otimes U^{*} \oplus S^{2} U^{*}$. Thus $U \otimes U^{*}=\mathfrak{h}$. Finally, since $\mathfrak{g}$ is completely determined by the $\mathfrak{h}$-module structure of $\mathfrak{g}_{1}$, we conclude that $\mathfrak{g}=\mathfrak{s l}_{m}$ for some integer $m$. Our proof of the classification of complex simple Lie algebras is now complete.

If we adopt the convention that $\tau(Y)$ is the quartic hypersurface $\left\langle u, u^{*}\right\rangle^{2}=0$, which is a double quadric, and $I_{3}\left(\tau(Y)_{\text {sing }}\right)$ is the space of derivatives of this quartic, then the adjoint algorithm also works in this case. (However usually one would take the tangential variety of $Y$ to be $Y$ itself.)

For example, for the adjoint variety of $\mathfrak{s l}_{3}$ (the projectivization of the space of matrices of order three, rank one and trace zero) we have the following quartic parametrization:

$$
(a, b, c, d) \mapsto\left(\begin{array}{ccc}
a^{3} d+a^{2} b c & -a^{2} b d-a b^{2} c & b^{2} c^{2}-a^{2} d^{2} \\
2 a^{3} c & -2 a^{2} b c & 2 a b c^{2}-2 a^{2} c d \\
a^{4} & -a^{3} b & a^{2} b c-a^{3} d
\end{array}\right) .
$$

\section{References}

[1] Bourbaki N., Groupes et algèbres de Lie, Hermann, Paris 1968.

[2] Bryant R.L., Chern S.S., Gardner R.B., Goldschmidt H.L., Griffiths P.A., Exterior differential systems, Publications M.S.R.I., Springer-Verlag 1991.

[3] Fulton W. Hansen J., A connectedness theorem for projective varieties, with applications, Ann. Math. 110, 159-166 (1970).

[4] Landsberg J.M., Differential-geometric characterizations of complete intersections, $J$. Diff. Geom. 44, 32-73 (1996).

[5] Landsberg J.M., On degenerate secant and tangential varieties and local differential geometry, Duke Math. J. 85, 605-634 (1996).

[6] Landsberg J.M.:, On the infinitesimal rigidity of homogeneous varieties, Compositio Math. 118, 189-201 (1999). 
[7] Landsberg J.M., Manivel L., On the projective geometry of homgeneous varieties, eprint math.AG/9810140.

[8] Landsberg J.M., Manivel L., The projective geometry of Freudenthal's magic square, $J$. Alg. 239, 477-512 (2001).

[9] Landsberg J.M., Manivel L., Series of Lie algebras, after Deligne, Freudenthal and Zak, in preparation.

[10] Landsberg J.M., Manivel L., Triality, exceptional Lie algebras and Deligne dimension formulas, eprint math.AG/0107032.

[11] Lichtenstein W., A system of quadrics describing the orbit of the highest weight vector, Proc. A.M.S. 84, 605-608 (1982).

[12] Onishchik A, Vinberg E., Lie groups and Lie algebras III, Encyclopaedia of Mathematical Sciences 41, 1994.

[13] Zak F., Tangents and Secants of Algebraic Varieties, AMS Translations of Mathematical Monographs 127, 1993.

Joseph M. Landsberg

Mathematics Department,

Georgia Institute of Technology,

Atlanta, GA 30332-0160

UNITED STATES

E-mail: jml@math.gatech.edu

Laurent Manivel

Institut Fourier, UMR 5582 du CNRS

Université Grenoble I

BP 74

38402 Saint Martin d'Hères cedex

FRANCE

E-mail: Laurent.Manivel@ujf-grenoble.fr 